\title{
On Harnack inequalities and singularities of admissible metrics in the Yamabe problem
}

\author{
Neil S. Trudinger · Xu-Jia Wang
}

Received: 12 April 2007 / Accepted: 11 September 2008 / Published online: 2 December 2008

C Springer-Verlag 2008

\begin{abstract}
In this paper we study the local behaviour of admissible metrics in the $k$-Yamabe problem on compact Riemannian manifolds $\left(M, g_{0}\right)$ of dimension $n \geq 3$. For $n / 2<k<n$, we prove a sharp Harnack inequality for admissible metrics when $\left(M, g_{0}\right)$ is not conformally equivalent to the unit sphere $S^{n}$ and that the set of all such metrics is compact. When $\left(M, g_{0}\right)$ is the unit sphere we prove there is a unique admissible metric with singularity. As a consequence we prove an existence theorem for equations of Yamabe type, thereby recovering as a special case, a recent result of Gursky and Viaclovsky on the solvability of the $k$-Yamabe problem for $k>n / 2$.
\end{abstract}

\section{Mathematics Subject Classification (2000) $\quad 53 \mathrm{C} 21 \cdot 58 \mathrm{~J} 05$}

\section{Introduction}

Let $\left(\mathcal{M}, g_{0}\right)$ be a compact Riemannian manifold of dimension $n \geq 3$ and $\left[g_{0}\right.$ ] the set of metrics conformal to $g_{0}$. For $g \in\left[g_{0}\right]$ we denote by

$$
A_{g}=\frac{1}{n-2}\left(\operatorname{Ric}_{g}-\frac{R_{g}}{2(n-1)} g\right)
$$

the Schouten tensor and by $\lambda\left(A_{g}\right)=\left(\lambda_{1}, \ldots, \lambda_{n}\right)$ the eigenvalues of $A_{g}$ with respect to $g$ (so one can also write $\lambda=\lambda\left(g^{-1} A_{g}\right)$ ), where Ric and $R$ are respectively the Ricci tensor and the scalar curvature. We also denote as usual

This work was supported by the Australian Research Council.

N. S. Trudinger $(\bowtie) \cdot$ X.-J. Wang

Centre for Mathematics and its Applications, Australian National University,

Canberra, ACT 0200, Australia

e-mail: neil.trudinger@maths.anu.edu.au

X.-J. Wang

e-mail: wang@maths.anu.edu.au 


$$
\sigma_{k}(\lambda)=\sum_{i_{1}<\cdots<i_{k}} \lambda_{i_{1}} \cdots \lambda_{i_{k}}
$$

the $k$ th elementary symmetric polynomial and

$$
\Gamma_{k}=\left\{\lambda \in \mathbf{R}^{n} \mid \sigma_{j}(\lambda)>0 \text { for } j=1, \ldots, k\right\}
$$

the corresponding open, convex cone in $\mathbf{R}^{n}$. Denote

$$
\left[g_{0}\right]_{k}=\left\{g \in\left[g_{0}\right] \mid \lambda\left(A_{g}\right) \in \bar{\Gamma}_{k}\right\} .
$$

Following [3], we call a metric in $\left[g_{0}\right]_{k} k$-admissible. In this paper we prove three main theorems pertaining to the cases $k>\frac{n}{2}$.

Theorem A If $\left(\mathcal{M}, g_{0}\right)$ is not conformally equivalent to the unit sphere $S^{n}$ and $\frac{n}{2}<k \leq n$, then $\left[g_{0}\right]_{k}$ is compact in $C^{0}$ and satisfies the following Harnack inequality, namely for any $g=\chi g_{0} \in\left[g_{0}\right]_{k}$,

$$
\max _{x, y \in \mathcal{M}} \frac{\chi(x)}{\chi(y)} \leq \exp \left(C|x-y|^{2-\frac{n}{k}}\right)
$$

for some fixed constant $C$ depending only on $n, k$ and $\left(\mathcal{M}, g_{0}\right)$, where $|x-y|$ denotes the geodesic distance in the metric $g_{0}$ between $x$ and $y$.

By compactness we mean in the sense of restriction to conformal metrics with fixed volume. Equivalently the compactness can be understood as the set of all conformal factors $\chi$ with $\sup \chi=1$. See also (3.28) below. When the manifold $\left(\mathcal{M}, g_{0}\right)$ is the unit sphere, the compactness is no longer true. In this case $\left(\mathcal{M}, g_{0}\right)$ with one point removed is conformally equivalent to the Euclidean space $\mathbf{R}^{n}$ so that without loss of generality, it suffices to study conformal metrics on $\mathbf{R}^{n}$. For our investigation we will allow singular metrics. Accordingly we call a metric $g=\chi g_{0} k$-admissible if $\chi: \mathcal{M} \rightarrow(0, \infty], \chi$ is lower semi-continuous, $\not \equiv \infty$ and there exists a sequence of $k$-admissible metrics $g_{m}=\chi_{m} g_{0}, \chi_{m} \in C^{2}(\mathcal{M})$, such that $\chi_{m} \rightarrow \chi$ almost everywhere in $\mathcal{M}$. If $g$ is $k$-admissible, then the function $v=\chi^{(n-2) / 4}$ is subharmonic with respect to the operator

$$
\square:=-\Delta_{g}+\frac{n-2}{4(n-1)} R_{g}
$$

and hence by the weak Harnack inequality $[11,25]$, the set $\{\chi=\infty\}$ has measure zero. Our next result classifies the possible singularities of $k$-admissible metrics on $\mathbf{R}^{n}$.

Theorem B Let $g$ be $k$-admissible on $\mathbf{R}^{n}$ with $\frac{n}{2}<k \leq n$. Then either

$$
g(x)=\frac{C}{\left|x-x_{0}\right|^{4}} g_{0}(x)
$$

for some point $x_{0} \in \mathbf{R}^{n}$ and positive constant $C$, or the conformal factor $\chi$ is Hölder continuous with exponent $\alpha=2-\frac{n}{k}$, where $g_{0}$ is the standard metric on $\mathbf{R}^{n}$.

Remark Theorems A and B also extend to metrics where the condition $g \in\left[g_{0}\right]_{k}$ (namely $\left.\lambda\left(A_{g}\right) \in \Gamma_{k}\right)$ is replaced by $P_{\delta}(\lambda)>0$, for some constant $\delta<1 /(n-2)$, where $P_{\delta}$ is the Pucci operator [11], given by,

$$
P_{\delta}(\lambda)=\min \lambda_{i}+\delta \sum \lambda_{i}
$$


By (1.1), the Ricci curvature $\mu=\left(\mu_{1}, \ldots, \mu_{n}\right)$ is given by

$$
\mu_{i}=(n-2) \lambda_{i}+\sum \lambda_{j}
$$

Hence the Ricci curvature $\mu_{i} \geq 0$ for all $1 \leq i \leq n$ if and only if $P_{\frac{1}{n-2}}(\lambda) \geq 0$. We remark that when $\lambda \in \Gamma_{k}$ and $k \geq \frac{n}{2}$, the Ricci curvature is nonnegative [15]. Indeed, for any $\lambda \in \Gamma_{k}$, let $f(x)=\frac{1}{2} \sum \lambda_{k} x_{k}^{2}$. By direct computation, $\Delta_{p} f \geq 0$ for $p \leq 2+\frac{n(k-1)}{n-k}$ (this is the simplest case, namely $l=1$, in Lemma 4.2 in [27]). Hence

$$
\sum \lambda_{i}+\frac{n(k-1)}{n-k} \lambda_{j} \geq 0
$$

for every $j$. Hence $\operatorname{Ric}_{g} \geq 0$ if $\lambda\left(A_{g}\right) \in \Gamma_{n / 2}$, and $\operatorname{Ric}_{g}>0$ if $\lambda\left(A_{g}\right) \in \Gamma_{k}$ for $k>\frac{n}{2}$.

Theorems A and B have various interesting consequences, in particular to the existence of conformal metrics with prescribed $k$-curvature. This is the problem of finding a conformal metric $g \in\left[g_{0}\right]$ such that

$$
\sigma_{k}\left(\lambda\left(A_{g}\right)\right)=f
$$

where $f$ is a given positive smooth function on $\mathcal{M}$. When $f \equiv 1,(1.9)$ is the $k$-Yamabe problem, which has been studied by many authors; see $[1,22,26]$ for $k=1$ and $[5,6,10,14$, $18,20,21,24,29]$ for $k \geq 2$. For $k>\frac{n}{2}$, it was recently resolved by Gursky and Viaclovski [18], by proving compactness results and characterizations of singularities for solutions. Our Theorems A and B above show that such results are already valid for admissible metrics, that is those in the conformal class $\left[g_{0}\right]$ in which solutions are sought. As well, Theorem A provides a sharp version of the solution estimates in [18].

By writing $g=v^{4 /(n-2)} g_{0}$, we see that (1.9) is equivalent to the conformal $k$-Hessian equation

$$
\sigma_{k}(\lambda(V))=\varphi(x, v)
$$

where

$$
V=-\nabla^{2} v+\frac{n}{n-2} \frac{\nabla v \otimes \nabla v}{v}-\frac{1}{n-2} \frac{|\nabla v|^{2}}{v} g_{0}+\frac{n-2}{2} v A_{g_{0}},
$$

$\lambda(V)$ denotes the eigenvalues of the matrix $V$, and $\varphi=f v^{k \frac{n+2}{n-2}}$. When $k \geq 2$, (1.10) is a fully nonlinear partial differential equation, which is elliptic if the eigenvalues $\lambda\left(A_{g}\right) \in \Gamma_{k}$. Therefore to study problem (1.9), we always assume $\left[g_{0}\right]_{k} \neq \varnothing$. Under this assumption, the $k$-Yamabe problem has been solved in [24] if $2 \leq k \leq \frac{n}{2}$ and (1.9) is variational. Equation (1.9) is automatically variational when $k=2$, but when $k \geq 3$, it is variational when the manifold is locally conformally flat. When $\frac{n}{2}<k \leq n$, the existence of solutions to (1.9) was proved in [18] for any smooth, positive function $f$; see also [6] for the solvability when $k=2$ and $n=4$, and $[14,20]$ when the manifold is locally conformally flat. For completeness and to illustrate the application of Theorem A, we prove the following extension.

Theorem $\mathbf{C}$ Let $\left(\mathcal{M}, g_{0}\right)$ be a compact $n$-manifold not conformally equivalent to the unit sphere $S^{n}$. Suppose $\frac{n}{2}<k \leq n$ and $\left[g_{0}\right]_{k} \neq \emptyset$. Then for any smooth, positive function $f$ and any constant $p \neq k$, there exists a positive admissible solution to the equation

$$
\sigma_{k}(\lambda(V))=f(x) v^{p} .
$$


The solution is unique if $p<k$. When $p=k$, then there exists a unique constant $\theta>0$ such that

$$
\sigma_{k}(\lambda(V))=\theta f(x) v^{k}
$$

has a solution, which is unique up to a constant multiplication.

We may call the constant $\theta$ in (1.13) (with $f \equiv 1$ ) the eigenvalue of the conformal $k$-Hessian operator in (1.10). As a special case of Theorem C, letting $p=k \frac{n+2}{n-2}$, we obtain the existence of solutions to the $k$-Yamabe problem (1.9) for $\frac{n}{2}<k \leq n$, as proved in [18]. As indicated above, the full strength of Theorem A is not necessary for these applications and solution estimates as in [18] would suffice. We also include some extensions of Theorem C at the end of Sect. 4.

As in [24] we will use conformal transformations of different forms,

$$
g=\chi g_{0}=v^{\frac{4}{n-2}} g_{0}=u^{-2} g_{0}=e^{-2 w} g_{0}
$$

so that

$$
u=v^{-2 /(n-2)}=e^{w} .
$$

We say $u, v$, or $w$ is conformally $k$-admissible, or simply $k$-admissible if no confusion arises, if the metric $g$ is $k$-admissible. In the smooth case, from the matrix $V$ in (1.11), we see that $u, w$ are $k$-admissible if the eigenvalues of the matrices

$$
\begin{aligned}
U & =\left\{u_{i j}-\frac{|D u|^{2}}{2 u} g_{0}+u A_{g_{0}}\right\}, \\
W & =\left\{w_{i j}+w_{i} w_{j}-\frac{1}{2}|D w|^{2} g_{0}+A_{g_{0}}\right\}
\end{aligned}
$$

lie in $\bar{\Gamma}_{k}$, the closure of $\Gamma_{k}$. Note that if $g$ is the metric given by (1.7), then

$$
v=\frac{C}{\left|x-x_{0}\right|^{n-2}}
$$

is the fundamental solution of the Laplace operator.

The conformal $k$-Hessian equation is closely related to the $k$-Hessian equation

$$
\sigma_{k}\left(\lambda\left(D^{2} u\right)\right)=\varphi \text { in } \Omega \text {, }
$$

where $\Omega \subset \mathbf{R}^{n}$ is a bounded domain. For the $k$-Hessian equation (1.19), it is proved in [27] that when $\frac{n}{2}<k \leq n$, a $k$-admissible function (relative to (1.19)) is locally Hölder continuous with Hölder exponent $\alpha=2-\frac{n}{k}$. The existence of solutions to (1.19) with right hand side $\varphi=f(x)|u|^{p}$ for some constant $p>0$ was studied in [9] for $k \leq \frac{n}{2}$ and in [8,30] for $k=n$. By the Hölder continuity one can extend the results in $[8,30]$ to the cases $\frac{n}{2}<k \leq n$. The argument in [30] uses a degree theory, which does not require a variational structure. We will employ the same degree argument to prove our Theorem C.

The proof of Theorem A can roughly be divided into two steps. In the first one we study the singularity behavior of admissible functions. Let $g_{m}=v_{m}^{\frac{4}{n-2}} g_{0}$ be a sequence of admissible metrics in $\left[g_{0}\right]_{k}$ for $k>\frac{n}{2}$. Then $v_{m} /$ inf $v_{m}$ converges in locally uniformly to an admissible function $v$. Moreover, if $x_{0}$ is a singular point of $v$, we prove that

$$
v(x)=\frac{C_{0}+o(1)}{\left|x-x_{0}\right|^{n-2}}
$$


where $C_{0}$ is a positive constant, $\left|x-x_{0}\right|$ denotes the geodesic distance from $x$ to $x_{0}$ in the metric $g_{0}$. In the second step we show that if (1.20) holds at all singular points, then by Bishop's volume growth formula, as used in [18], $w$ is smooth away from $x_{0}$, and the manifold $\left(\mathcal{M}, g_{0}\right)$ is conformally equivalent to the unit sphere.

The asymptotic behavior (1.20) has been established for the blow-ups of solutions to the classical Yamabe problem. Also a weak form of (1.20) was proved by Gursky and Viaclovsky [18], namely if $g_{m}=v_{m}^{\frac{4}{n-2}} g_{0}$ is a sequence of solutions to (1.5) for $k>\frac{n}{2}$, then $v_{m} / \inf v_{m}$ converges locally uniformly away from finitely many singular points to an admissible function $v$, and $v$ satisfies

$$
\frac{C_{1}}{\left|x-x_{0}\right|^{n-2}} \leq v(x) \leq \frac{C_{2}}{\left|x-x_{0}\right|^{n-2}}
$$

at any singular point $x_{0}$. From (1.21) they proved that $\left(\mathcal{M}, g_{0}\right)$ is conformally equivalent to the unit sphere. Basically our proof in the second step is inspired by that in [18], however our proof is somewhat simpler due to the stronger asymptotic estimate (1.20).

In this paper we also introduced a new technique. That is we reduce the singularity analysis of a $k$-admissible function to its maximal radial function. Given an admissible function $v$, we say $\tilde{v}$ is the maximal radial function of $v$ with center at $x_{0}$ if $\tilde{v}$ is a rotationally symmetric with respect to $x_{0}, \tilde{v} \leq v$, and $\tilde{v} \geq \varphi$ for any radial function $\varphi$ satisfying $\varphi \leq v$. Obviously $\tilde{v}$, as a function of $\left|x-x_{0}\right|$, is monotone increasing, namely $\tilde{v}(r) \geq \tilde{v}\left(r^{\prime}\right)$ for any $0<r^{\prime}<r$. We reduce the proof of (1.20) for $v$ to that for $\tilde{v}$. This new technique also applies to other nonlinear elliptic equations, which will be shown in a subsequent paper [28].

This paper is arranged as follows. In Sect. 2 we first prove Theorem B for radially symmetric, $k$-admissible functions defined on $\mathbf{R}^{n}$, then extend it to general $k$-admissible functions by considering the corresponding minimal radial function for $w=-\frac{2}{n-2} \log v$. In Sect. 3 we prove Theorem A. By studying the corresponding minimal radial function, we show that if an admissible function $w$ is a $k$-admissible function on a manifold $\mathcal{M}$, then either $w$ is Hölder continuous, or (1.20) holds at some singular point $x_{0} \in \mathcal{M}$. We then show that if (1.20) occurs, then $w$ is smooth away from $x_{0}$ and the manifold $\mathcal{M}$ is conformally equivalent to the unit sphere. In Sect. 4 we prove Theorem $\mathrm{C}$ by a degree argument.

In a subsequent paper [28], we will establish the asymptotic behavior (1.20) for any blow-up functions of solutions to the $k$-Yamabe problem (for $1 \leq k \leq \frac{n}{2}$ ) and the problem of prescribing more general symmetric curvature functions. In particular we prove the existence and compactness of solutions to the $k$-Yamabe problem for $k=\frac{n}{2}$.

\section{Proof of Theorem B}

\subsection{Radial functions}

We first demonstrate our idea by considering radially symmetric functions. Let $w$ be a radially symmetric, $k$-admissible function on $\mathbf{R}^{n} \backslash\{0\}$. For any given point $x \neq 0$, by a rotation of axes we assume $x=(0, \ldots, 0, r)$. Regard $w$ as a function of $r=|x|, r \in(0, \infty)$. Then the matrix $W$ in (1.17) is diagonal,

$$
W=\operatorname{diag}\left(\frac{1}{r} w^{\prime}-\frac{1}{2} w^{\prime 2}, \ldots, \frac{1}{r} w^{\prime}-\frac{1}{2} w^{\prime 2}, w^{\prime \prime}+\frac{1}{2} w^{\prime 2}\right)
$$


Denote $a=w^{\prime \prime}+\frac{1}{2} w^{\prime 2}$ and $b=\frac{1}{r} w^{\prime}-\frac{1}{2} w^{\prime 2}$. We have

$$
\begin{aligned}
\sigma_{k}(\lambda(W)) & =b^{k} C_{n-1}^{k}+a b^{k-1} C_{n-1}^{k-1} \\
& =C_{n-1}^{k-1} b^{k-1}\left(a+\frac{n-k}{k} b\right) .
\end{aligned}
$$

Since $\lambda(W) \in \bar{\Gamma}_{k}$ and $k>\frac{n}{2}$,

$$
\begin{aligned}
b & =\frac{w^{\prime}}{r}-\frac{1}{2} w^{\prime 2} \geq 0, \\
a+\frac{n-k}{k} b & =\left(w^{\prime \prime}+\frac{w^{\prime}}{r}\right)-(1-\theta)\left(\frac{w^{\prime}}{r}-\frac{1}{2} w^{\prime 2}\right) \geq 0,
\end{aligned}
$$

where $\theta=\frac{n-k}{k}<1$. It follows that

$$
\begin{aligned}
& 0 \leq w^{\prime} \leq \frac{2}{r}, \\
& w^{\prime \prime}+\frac{w^{\prime}}{r} \geq 0 .
\end{aligned}
$$

By sub-harmonicity of $w$, we have $w^{\prime}(r)=0$ for $r \in\left(0, r_{0}\right)$ if $w^{\prime}\left(r_{0}\right)=0$. Note that (2.5) can also be written as $\left(r w^{\prime}\right)^{\prime} \geq 0$. Therefore we have

Lemma 2.1 The function $r w^{\prime}$ is nonnegative, monotone increasing, and $r w^{\prime} \leq 2$.

It follows that $w$ must be locally uniformly bounded from above, namely if $w\left(r_{0}\right) \leq 0$, then $w \leq C$ for some constant $C$ depending only on $r_{0}$. Next we prove

Lemma 2.2 The function $w$ is either Hölder continuous in $\mathbf{R}^{n}$ with exponent $\alpha=2-\frac{n}{k}$, or

$$
w(r)=2 \log r+C
$$

for some constant $C$.

Proof It suffices to prove that $w$ is either Hölder continuous near $r=0$ or (2.6) holds. If $w$ is not Hölder continuous near $r=0$, then $w^{\prime}>0$ as $v=e^{\frac{n-2}{2} w}$ is superharmonic with respect to the operator (1.6).

If $r w^{\prime} \not \equiv 2$, then by Lemma $2.1, \lim _{r \rightarrow 0} r w^{\prime}=c_{0}<2$. For any $c_{1} \in\left(c_{0}, 2\right)$,

$$
w^{\prime \prime}+\frac{w^{\prime}}{r} \geq(1-\theta) \frac{w^{\prime}}{r}\left(1-\frac{1}{2} r w^{\prime}\right) \geq(1-\theta)\left(1-\frac{c_{1}}{2}\right) \frac{w^{\prime}}{r}
$$

if $r$ is sufficiently small. Hence

$$
\frac{w^{\prime \prime}}{w^{\prime}}+\frac{\sigma}{r} \geq 0
$$

where $\sigma=1-(1-\theta)\left(1-\frac{c_{1}}{2}\right)<1$. We obtain

$$
\left.\log \left(w^{\prime} r^{\sigma}\right)\right|_{r} ^{r_{0}} \geq 0
$$

Hence

$$
w^{\prime} \leq \frac{C}{r^{\sigma}} .
$$

Hence $w$ is bounded and continuous. 
To show that $w$ is Hölder continuous with Hölder exponent $\alpha=2-\frac{n}{k}$, by Lemma 2.1 it suffices to prove it at $r=0$. Note that

$$
a+\theta b=w^{\prime \prime}+\theta \frac{w^{\prime}}{r}+\frac{1-\theta}{2} w^{\prime 2} \geq 0 .
$$

Hence

$$
\frac{w^{\prime \prime}}{w^{\prime}}+\frac{\theta}{r} \geq-\frac{1-\theta}{2} w^{\prime}
$$

Taking integration from $r$ to $r_{0}$, we obtain

$$
\left.\log \left(w^{\prime} r^{\theta}\right)\right|_{r} ^{r_{0}} \geq C
$$

Hence

$$
w^{\prime} \leq \frac{C}{r^{\theta}},
$$

so that $w$ is Hölder continuous with exponent $1-\theta=2-\frac{n}{k}$.

Remark 2.1 The Hölder continuity also follows from [27]. Let $u=e^{w}$ be as in (1.15). From the matrix $U$ in (1.16), one sees that $u$ is $k$-admissible with respect to the $k$-Hessian operator $\sigma_{k}\left(\lambda\left(D^{2} u\right)\right)$. To show that $u$ is Hölder continuous with exponent $\alpha=2-\frac{n}{k}$, one applies the comparison principle to $u$ and $\varphi(x)=C|x-y|^{2-n / k}+u(y)$ (relative to the $k$-Hessian equation), where $C$ is chosen such that $u \leq \varphi$ on $\partial B_{R}(y)$, ones sees that $u(x)-u(y) \leq \varphi(x)$ in $B_{R}(y)$.

It follows that for any constant $c>0, w_{c}=\max (w,-c)$ is also Hölder continuous with exponent $2-\frac{n}{k}$. In particular, if $w_{m}$ converges to $w$ a.e., then $w_{m}$ converges to $w$ uniformly in $\{w>-c\}$ for any $c>0$.

\subsection{Proof of Theorem B}

Let $w$ be a $k$-admissible function. For any $h \in \mathbf{R}$, denote $\Omega_{h}=\{w<h\}$. Since $w$ is upper semi-continuous, $\Omega_{h}$ is an open set. For any given point 0 , we define a function $\tilde{w}$ of one variable $r$ by

$$
\tilde{w}(r)=\inf \left\{h: \operatorname{dist}\left(0, \partial \Omega_{h}\right)>r\right\} .
$$

We call $\tilde{w}$ the minimal radial function of $w$ (with respect to 0 ). It is a function of $|x|$, satisfying $\tilde{w} \geq w$ and $\tilde{w} \leq \varphi$ for any radial function $\varphi$ satisfying $\varphi \geq w$. Obviously $\tilde{w}$ is monotone increasing, namely $\tilde{w}(r) \geq \tilde{w}\left(r^{\prime}\right)$ for any $0<r^{\prime}<r$.

Let $x_{h} \in \partial \Omega_{h}$ such that $\left|x_{h}\right|=r_{h}:=\operatorname{dist}\left(0, \partial \Omega_{h}\right)$. Assume that $\partial \Omega_{h}$ and $w$ are smooth at $x_{h}$. Rotate the axes such that $x_{h}=\left(0, \ldots, 0, r_{h}\right)$. Then the $x_{n}$-axis is the outer normal of $\partial \Omega_{h}$ at $x_{h}$. Hence

$$
\begin{aligned}
\tilde{w}\left(r_{h}\right) & =w\left(x_{h}\right), \\
\tilde{w}\left(r_{h}+t\right) & \geq w\left(x_{h}+t e_{h}\right)
\end{aligned}
$$

for $t$ near 0 , where $e_{n}=(0, \ldots, 0,1)$. We obtain

$$
\begin{aligned}
\tilde{w}^{\prime}\left(r_{h}\right) & =w_{n}\left(x_{h}\right)=|D w|\left(x_{h}\right), \\
\tilde{w}^{\prime \prime}\left(r_{h}\right) & \geq w_{n n}\left(x_{h}\right)
\end{aligned}
$$

provided $\tilde{w}$ is twice differentiable point at $r_{h}$. 
Let $\kappa_{1}, \ldots, \kappa_{n-1}$ be the principal curvatures of $\partial \Omega_{h}$ at $x_{h}$. Then, after a rotation of the axes $\left(x_{1}, \ldots, x_{n-1}\right)$,

$$
w_{i j}=|D w| \kappa_{i} \delta_{i j} \quad i, j \leq n-1 .
$$

By our choice of $x_{h}$, we have

$$
\kappa_{i} \leq \frac{1}{r}
$$

where $r=r_{h}$. Hence the matrix

$$
\left(w_{i j}\right)_{i, j=1}^{n-1} \leq \frac{1}{r}|D w| I .
$$

At $x_{h}$, the matrix $W$ is given by

$$
\begin{aligned}
W & =\left\{w_{i j}+w_{i} w_{j}-\frac{1}{2}|D w|^{2} I\right\} \\
& =\left(\begin{array}{lll}
w_{11}-\frac{1}{2}|D w|^{2}, & 0, & \cdots, w_{1 n} \\
0, & w_{22}-\frac{1}{2}|D w|^{2}, & \cdots, w_{2 n} \\
\cdot & \cdot & \cdot \\
\cdot & \cdot & \cdot \\
w_{1 n}, & \cdots, w_{n n}+\frac{1}{2}|D w|^{2}
\end{array}\right)
\end{aligned}
$$

Let

$$
\hat{W}=\operatorname{diag}\left(w_{11}-\frac{1}{2}|D w|^{2}, w_{22}-\frac{1}{2}|D w|^{2}, \ldots, w_{n n}+\frac{1}{2}|D w|^{2}\right)
$$

be a diagonal matrix. By Lemma 2.3 below, the eigenvalues $\lambda(\hat{W}) \in \bar{\Gamma}_{k}$.

Lemma 2.3 Let $A=\left(a_{i j}\right)$ be an $n \times n$ symmetric matrix with eigenvalues $\lambda(A) \in \Gamma_{k}$. Then $\lambda(\hat{A})) \in \Gamma_{k}$, where $\hat{A}=\left(\begin{array}{ll}A^{\prime}, & 0 \\ 0, & a_{n n}\end{array}\right), A^{\prime}=\left(a_{i j}\right)_{1 \leq i, j \leq n-1}$.

In particular if $\lambda(A) \in \Gamma_{k}$, then the vector $\left(a_{11}, \ldots, a_{n n}\right) \in \Gamma_{k}$.

Proof It suffices to prove $\sigma_{j}(\lambda(\hat{A})) \geq 0$ for all $j=1, \ldots, k$. Let us prove it for $j=k$. Recalling that $\sigma_{k}(\lambda(A))$ is the sum of all principal $k \times k$ minors, we have

$$
\sigma_{k}(\lambda(A))=\sigma_{k}(\lambda(\hat{A}))-\sum_{i<n} \sigma_{k-2}\left(\lambda\left(A_{\mid i n}\right)\right) a_{i n}^{2},
$$

where $A_{\mid i j}$ denotes the matrix obtained by canceling the $i$ th row and $j$ th column of $A$. Since $\lambda(A) \in \bar{\Gamma}_{k}$, we have

$$
\sigma_{k-2}\left(\lambda\left(A_{\mid i n}\right)\right)=\frac{\partial^{2} \sigma_{k}(\lambda(A))}{\partial a_{i i} \partial a_{n n}} \geq 0 .
$$

Hence $\sigma_{k}(\lambda(\hat{A})) \geq \sigma_{k}(\lambda(A)) \geq 0$.

From (2.15),

$$
\hat{W} \leq \operatorname{diag}\left(\frac{1}{r} \tilde{w}^{\prime}-\frac{1}{2}\left(\tilde{w}^{\prime}\right)^{2}, \ldots, \frac{1}{r} \tilde{w}^{\prime}-\frac{1}{2}\left(\tilde{w}^{\prime}\right)^{2}, \tilde{w}^{\prime \prime}+\frac{1}{2}\left(\tilde{w}^{\prime}\right)^{2}\right) .
$$


Therefore as in Sect. 2.1, we see that $\tilde{w}$ satisfies

$$
\begin{aligned}
& \frac{\tilde{w}^{\prime}}{r}-\frac{1}{2}\left(\tilde{w}^{\prime}\right)^{2} \geq 0 \\
& \left(\tilde{w}^{\prime \prime}+\frac{\tilde{w}^{\prime}}{r}\right)-(1-\theta)\left(\frac{\tilde{w}^{\prime}}{r}-\frac{1}{2}\left(\tilde{w}^{\prime}\right)\right)^{2} \geq 0
\end{aligned}
$$

if $\tilde{w}$ is twice differentiable at $r$.

To proceed further we make a remark.

Remark 2.2 Inequalities (2.20) and (2.21) are exactly (2.2) and (2.3). In the present case, the function $\tilde{w}$ may not be smooth. But by definition, $w$ can be approximated by smooth $k$-admissible functions. Hence $\tilde{w}$ can be approximated by piecewise smooth functions, which satisfy $\lim _{r \rightarrow r_{0}^{-}} \tilde{w}^{\prime}(r) \leq \lim _{r \rightarrow r_{0}^{+}} \tilde{w}^{\prime}(r)$ by $(2.11)$.

For a piecewise smooth function $w$ with nonsmooth points $r_{1}>r_{2} \cdots>r_{j}>\cdots$ at which $\lim _{r \rightarrow r_{j}^{-}} \tilde{w}^{\prime}(r)<\lim _{r \rightarrow r_{j}^{+}} \tilde{w}^{\prime}(r)$, we can mollify $w$ at the points $r_{j}$ to get a smooth function $w^{*}$ which satisfies $\lim _{r \rightarrow 0} w^{*}(r)=\lim _{r \rightarrow 0} w(r)$, and also satisfies (2.20) and (2.21). The proof of Lemma 2.2 implies that if $\lim _{r \rightarrow 0} w^{*}(r)=-\infty$, then $r\left(w^{*}\right)^{\prime} \equiv 2$; if $\lim _{r \rightarrow 0} w^{*}(r)>-\infty$, then $w^{*}$ is Holder continuous with its Holder norm independent of the mollification.

We can now prove Theorem B easily. First we consider the case when $w$ is unbounded from below.

Lemma 2.4 Let $w$ be a $k$-admissible function which is locally unbounded from below, then there exists a point $x_{0} \in \mathbf{R}^{n}$ and a constant $C$ such that

$$
w(x) \equiv 2 \log \left|x-x_{0}\right|+C .
$$

Proof If $w$ is locally unbounded from below, the singular set $S=\bigcap_{\{c<0\}}\{w<c\}$ is not empty. Choose a point $0 \in S$. By (2.20) and (2.21), and from the argument in Sect. 2.1, we must have $\tilde{w}(r)=2 \log r+C$ for some constant $C$.

Let $\hat{w}=2 \log |x|+C$. Then

$$
\begin{aligned}
& \sigma_{1}\left(\lambda\left(W_{\hat{w}}\right)\right)=0, \\
& \sigma_{1}\left(\lambda\left(W_{w}\right)\right) \geq \sigma_{k}^{1 / k}\left(\lambda\left(W_{w}\right)\right) \geq 0,
\end{aligned}
$$

where $W_{\hat{w}}$ is the matrix corresponding to $\hat{w}$, given in (1.17). Note that $\sigma_{1}(\lambda(W))$ is indeed the Laplace operator in $\mathbf{R}^{n}$ (in terms of $v$, by the relation (1.15)). By our definition of $\tilde{w}$, we have $\hat{w} \geq w$. Since $\tilde{w}=2 \log r+C$, we see that $w-\hat{w}$ attains its local maximum 0 at some interior point. By the maximum principle for the Laplace equation, we conclude that $w \equiv \hat{w}$.

Next we consider the case when $w$ is bounded from below.

Lemma 2.5 Let $w$ be a $k$-admissible function $w$. Suppose $w$ is bounded from below. Then $w$ is Hölder continuous with exponent $\alpha=2-\frac{n}{k}$.

Proof For any given point $x_{0}$, we may take $x_{0}$ as the origin and let $\tilde{w}$ be the minimal radial function of $w$ with respect to $x_{0}$, as defined in (2.10). Then to prove that $w$ is Hölder continuous at $x_{0}$ with exponent $\alpha=2-\frac{n}{k}$, it suffices to show that $\tilde{w}$ is Hölder continuous with exponent $\alpha$. But by (2.20), (2.21), the Hölder continuity of $\tilde{w}$ readily follows from the argument in Sect. 2.1, see (2.9). 
The Hölder continuity also follows from Remark 2.1 above.

Note that the function $w=2 \log |x|$ is $k$-admissible. By truncating at $w=-K$ (for large $K$ ), we see that the set of Hölder continuous $k$-admissible functions is not compact.

\subsection{Applications}

First we remark that, by the above proof, Theorem B also holds for $k$-admissible functions defined on a domain. Here we restate the theorem for the function $v=e^{-\frac{n-2}{2} w}$. Note that by Lemma $2.1, w$ is locally uniformly bounded, and so $v$ is locally strictly positive when $k>\frac{n}{2}$.

Theorem $\mathbf{B}^{\prime}$ Let $\Omega$ be a domain in $\mathbf{R}^{n}$. Let $v$ be a $k$-admissible function in $\Omega$ with $\frac{n}{2}<k \leq n$. If $v$ is unbounded from above near some point $x_{0} \in \Omega$, then

$$
v(x)=C\left|x-x_{0}\right|^{2-n} .
$$

Otherwise $v$ is locally Hölder continuous in $\Omega$ with exponent $\alpha=2-\frac{n}{k}$.

It was proved in [20] that if $v$ is a $k$-admissible function, so is the function $v_{\psi}$ in a punctured small ball $B_{r}(0) \backslash\{0\}$, where

$$
v_{\psi}=\left|J_{\psi}\right|^{\frac{n-2}{2 n}} v \circ \psi
$$

$\psi(x)=\frac{x}{|x|^{2}}$, and $J_{\psi}$ is the Jacobian of the mapping $\psi$. From Theorem $\mathrm{B}^{\prime}$ we have

Corollary 2.5 Let $v$ be a $k$-admissible function defined in $\mathbf{R}^{n} \backslash B_{1}(0)$ with $\frac{n}{2}<k \leq n$. Then either $v \equiv$ constant or $|x|^{n-2} v(x)$ converges to a positive constant as $x \rightarrow \infty$.

Proof We cannot apply Theorem $\mathrm{B}^{\prime}$ directly, as the function $v_{\psi}$ has a singular point at 0 . Denote $w=\frac{-2}{n-2} \log v_{\psi}$. If $w(x) \rightarrow-\infty$ as $x \rightarrow 0$, the argument in Sect. 2.2 implies that $w=2 \log |x|+C$. Changing back to $v$, we obtain $v \equiv$ constant. Otherwise it suffices to show that $w$ is continuous at 0 .

Let $w(0)=\varlimsup_{x \rightarrow 0} w(x)$ so that $w$ is upper semi-continuous. If $a:=\underline{\lim }_{x \rightarrow 0} w(x)<$ $w(0)$, by subtracting a constant we assume $w(0)=0$. Let $x_{m} \rightarrow 0$ such that $w\left(x_{m}\right)=-\frac{a}{2}$. Let $\tilde{w}=\tilde{w}_{x_{m}}$ be the minimal radial function of $w$ with respect to $x_{m}$, as defined in (2.10). We claim that when $m$ is sufficiently large, the point $x_{h}$ in (2.11) at $h=0$ cannot be the origin. In other words, there is a point $y_{m}$ with $w\left(y_{m}\right)=0$ such that $\left|y_{m}-x_{m}\right|<\left|x_{m}\right|$. Indeed, if $x_{h}=0$, by the Hölder continuity of $w$ in $B_{r_{m}}\left(x_{m}\right)$, where $r_{m}=\left|x_{m}\right|$ (Remark 2.1), we see that $w(x) \leq-\frac{a}{2}$ in $B_{\delta r_{m}}\left(x_{m}\right)$, for some $\delta>0$ independent of $m$. Namely $v>e^{\frac{(n-2) a}{4}}>1$ in $B_{\delta r_{m}}\left(x_{m}\right)$, where $v=v_{\psi}$. Recall that $\underline{\lim }_{x \rightarrow 0} v(x) \geq 1$, by the mean value inequality for super-harmonic function, we have

$$
v(0) \geq \frac{1}{\left|B_{2 r_{m}}\right|(0)} \int_{B_{2 r_{m}}(0)} v(x) d x>0 .
$$

We reach a contradiction.

When the singular point $x=0$ is not the extreme point $x_{h}$ in (2.11), the argument in Sect. 2.2 applies to $\tilde{w}=\tilde{w}_{x_{m}}$ in $B_{1}(0)$. In particular $\tilde{w}$ is uniformly Hölder continuous. Hence if $w(0)=0$ and $w\left(x_{m}\right) \leq-1$, we have $\left|x_{m}\right| \geq c_{0}>0$ for some $c_{0}$ independent of $m$. This is again a contradiction. Hence $w$ is continuous at 0 , and so $|x|^{n-2} v(x)$ converges to a positive constant as $x \rightarrow \infty$. 
Theorem B also implies the non-existence of solutions to the Dirichlet problem in general. Let $\Omega$ be a bounded domain in $\mathbf{R}^{n}$ which is not a ball. Then if $k>\frac{n}{2}$, there is no solution to the Dirichlet problem

$$
\begin{aligned}
\sigma_{k}(\lambda(V)) & =f \quad \text { in } \quad \Omega, \\
v & =c \quad \text { on } \quad \partial \Omega
\end{aligned}
$$

in general, where $c$ is any positive constant, and $f$ is a positive smooth function. Indeed, let $\left\{f_{m}\right\}$ be a sequence of smooth, positive functions which converges to zero locally uniformly in $\Omega \backslash\left\{x_{0}\right\}$ such that $\sup v_{m} \rightarrow \infty$, where $v_{m}$ is the corresponding solution (if the solution $v_{m}$ does not exist, we are through). Then $v_{m}$ must converge to the function $v=C\left|x-x_{0}\right|^{2-n}$ by Theorem B. Hence $\Omega$ must be a ball centered at $x_{0}$.

For the existence of solutions to the Dirichlet problem, it was proved recently by Guan [12] that for any smooth, bounded domain with smooth boundary data, the Dirichlet problem is classically solvable if there exists a smooth sub-solution with the same boundary trace.

\section{Proof of Theorem A}

\subsection{Hölder continuity}

We start with a Hölder continuity property of $k$-admissible functions.

Lemma 3.1 Let $\left(\mathcal{M}, g_{0}\right)$ be a compact manifold. Suppose $g=u^{-2} g_{0} \in\left[g_{0}\right]_{k}$ and $k>\frac{n}{2}$. Then $u$ is Hölder continuous with exponent $\alpha=2-\frac{n}{k}$,

$$
\frac{u(x)-u(y)}{|x-y|^{\alpha}} \leq C \int_{\mathcal{M}} u,
$$

where $C$ is independent of $u$.

Proof By approximation it suffices to prove (3.1) for smooth functions. For any given point $0 \in \mathcal{M}$, there exists a conformal normal metric $[2,4,16]$, still denoted by $g_{0}$, such that in the normal coordinates at 0 ,

$$
\operatorname{det}\left(g_{0}\right)_{i j} \equiv 1 \text { near } 0 \text {. }
$$

Let

$$
u_{0}(x)=|x|^{2-\frac{n}{k}}
$$

where $|x|$ denotes the geodesic distance from 0. Note that under condition (3.2), the Laplacian $\Delta$ on $\mathcal{M}$ is equal to the Euclidean Laplacian when applying to functions of $r=|x|$ alone $[19,23]$. Hence

$$
\Delta_{g_{0}} u_{0}=\frac{n(k-1)(2 k-n)}{k^{2}} r^{-\frac{n}{k}} .
$$

Denote by

$$
P[u]=\min \lambda_{i}+\delta \sum_{i} \lambda_{i}, \quad\left(\delta=\frac{n-k}{n(k-1)}\right)
$$


the Pucci minimal operator [11], where $\left(\lambda_{1}, \ldots, \lambda_{n}\right)$ are the eigenvalues of the Hessian matrix $\left(\nabla_{i j} u_{0}\right)$. Obviously we have

$$
\min \lambda_{i} \leq \partial_{r}^{2} u_{0}=-\frac{(2 k-n)(n-k)}{k^{2}} r^{-\frac{n}{k}} .
$$

Therefore $u_{0}$ satisfies

$$
P\left[u_{0}\right] \leq 0 \text { in } B_{0, r} \backslash\{0\} .
$$

where $B_{y, r}$ denotes the geodesic ball with center $y$ and radius $r$.

On the other hand, since $\lambda(U) \in \bar{\Gamma}_{k}$, where $U$ is given in (1.16), we have $\lambda\left(u_{i j}+u A_{g_{0}}\right) \in$ $\bar{\Gamma}_{k} \subset \bar{\Gamma}_{1}$. Namely $\Delta u+\operatorname{tr}\left(A_{g_{0}}\right) u \geq 0$. By the weak Harnack inequality [11] it follows

$$
\sup u \leq C \int_{\mathcal{M}} u,
$$

where $C$ is a constant independent of $u$. Therefore to prove (3.1) we may assume that $\int_{\mathcal{M}} u=1$ and $u$ is uniformly bounded.

Let $u_{a}=u+a|x|^{2}$. Then $\nabla^{2} u_{a}>\nabla^{2} u+a I$ near 0 , where $I$ is the unit matrix. Since $\lambda\left(\nabla^{2} u+u A_{g_{0}}\right) \in \bar{\Gamma}_{k}$, we have $\lambda\left(\nabla^{2} u_{a}\right) \in \Gamma_{k}$ when $a$ is suitably large. Taking $l=1$ in the proof of Lemma 4.2 in [27], one has

$$
\lambda_{i}+\frac{n-k}{n(k-1)} \sum_{i} \lambda_{i} \geq 0,
$$

namely $P\left[u_{a}\right] \geq 0$ near 0 . Hence by applying the comparison principle to the functions $u_{a}$ and $u_{0}$ with respect to the operator $P$, we conclude the Hölder continuity (3.1).

Remark 3.1 The estimate (3.1) (with exponent $\alpha<2-\frac{n}{k}, k>\frac{n}{2}$ ) also follows from gradient estimates from our reduction to $p$-Laplacian subsolution in [27]. Since $\lambda(U) \in \Gamma_{k}$, we have $\lambda\left(D^{2} u+u A_{g_{0}}\right) \in \Gamma_{k}$. By (3.8) it follows that

$$
\Delta_{p} u:=\nabla_{i}\left(|\nabla u|^{p-2} \nabla_{i} u\right) \geq-C u|\nabla u|^{p-2}
$$

for $p-2=\frac{n(k-1)}{n-k}$ and some constant $C$. From our argument in [27], we obtain $\int_{\mathcal{M}}|\nabla u|^{q} \leq C$ for any $q<n k /(n-k)$, whence by the Sobolev inequality, we infer (3.1) for $\alpha<2-\frac{n}{k}$; (see also [17]).

By the relation $u=e^{w}$, we have the following

Corollary 3.2 Let $w$ be a $k$-admissible function. Suppose $w \leq 0$. Then for any $K>0$, there exists $C=C_{K}>0$, independent of $w$, such that when $w(y)>-K$,

$$
\frac{w(x)-w(y)}{|x-y|^{\alpha}} \leq C
$$

with the same $\alpha$ as above.

From (3.10), we see that if $w(x) \leq-K-1$, then $|x-y| \geq C_{K+1}^{1 / \alpha}$. Also note that in Corollary 3.2, if we assume that $w \leq 0$ in $B_{y, r}$, then (3.10) holds for $x, y \in B_{y, r / 2}$ for some $C$ depending on $r$. 
3.2 Singularity behaviour of $k$-admissible functions

Suppose $w$ is a $k$-admissible function. At any given point $0 \in \mathcal{M}$, we choose a conformal normal coordinate near 0 such that (3.2) holds. In the conformal metric, the Ricci curvature vanishes at $0[19,23]$. Hence

$$
\left|A_{g_{0}}(x)\right| \leq C r \text { near } 0,
$$

where $r$ is the geodesic distance from 0 . Let $\tilde{w}$ be the minimal radial function of $w$ with respect to 0 , as defined in (2.10). Then the argument thereafter is still valid, except that (2.14) should be replaced by $\kappa_{i} \leq \frac{1}{r}+C$. Also note that in the conformal normal coordinate, at the point $(0, \ldots, 0, r)$ in the exponential map, the metric $g_{0}=I+J$, where $I$ is the unit matrix, $|J|=O(r)$ and its $n$ th-row and $n$ th-column vanishes. Hence from (2.19), we have

$$
(\tilde{b}, \ldots, \tilde{b}, \quad \tilde{a}) \in \bar{\Gamma}_{k},
$$

where

$$
\begin{aligned}
& \tilde{b}=\left(\frac{1}{r}+C\right) \tilde{w}^{\prime}-\frac{1-C r}{2}\left(\tilde{w}^{\prime}\right)^{2}+C r, \\
& \tilde{a}=\tilde{w}^{\prime \prime}+\frac{1}{2}\left(\tilde{w}^{\prime}\right)^{2}+C r .
\end{aligned}
$$

Similarly to the argument in Sect. 2.1 , we have $\tilde{b} \geq 0$ and $\tilde{a}+\frac{n-k}{k} \tilde{b} \geq 0$. Note that $\tilde{b} \geq 0$ is equivalent to that $\frac{1}{2}\left(\tilde{w}^{\prime}\right)^{2} \leq\left(\frac{1}{r}+C\right) \tilde{w}^{\prime}+C r$. Hence

$\tilde{a}+\frac{n-k}{k} \tilde{b} \geq\left[\tilde{w}^{\prime \prime}+\left(\frac{1}{r}+C\right) \tilde{w}^{\prime}+C r\right]-(1-\theta)\left[\left(\frac{1}{r}+C\right) \tilde{w}^{\prime}-\frac{1}{2}\left(\tilde{w}^{\prime}\right)^{2}+C r\right] \geq 0$, where $\theta=\frac{n-k}{k}$ is as in (2.3). It follows that, when $r>0$ is small,

$$
\begin{aligned}
& \tilde{w}^{\prime} \leq \frac{2}{r}+\frac{C r}{\tilde{w}^{\prime}}+C, \\
& \tilde{w}^{\prime \prime}+\left(\frac{1}{r}+C\right) \tilde{w}^{\prime}+C r \geq 0 .
\end{aligned}
$$

From (3.13),

$$
\tilde{w}^{\prime} \leq \frac{2}{r}+C
$$

for a different $C$. It follows that $\tilde{w}(r)>-\infty$ for any $r>0$, namely the set $\{w=-\infty\}$ cannot an open set containing the origin 0. But this property also follows from the weak Harnack inequality for the operator (1.6), as noted there.

From (3.14), we therefore obtain

$$
\left(r \tilde{w}^{\prime}\right)^{\prime}+C \geq 0 .
$$

It follows that $r \tilde{w}^{\prime}+C r$ is increasing. By the compactness of $\mathcal{M}$, a $k$-admissible function $w$ must be bounded from above.

If $r \tilde{w}^{\prime}<2$ near $r=0$, then similarly to (2.7) (2.8), $\tilde{w}$ is bounded and Hölder continuous.

If $r \tilde{w}^{\prime} \rightarrow 2$ as $r \rightarrow 0$, then $r \tilde{w}^{\prime}+C r \geq 2$, namely $\tilde{w}^{\prime} \geq \frac{2}{r}-C$. Hence we obtain

$$
\frac{2}{r}+C \geq \tilde{w}^{\prime} \geq \frac{2}{r}-C \text {. }
$$


We obtain

$$
\tilde{w}(r)=2 \log r+C^{\prime}+O(r) .
$$

By subtracting a constant we assume that $C^{\prime}=0$.

Lemma 3.3 If $\tilde{w}$ satisfies (3.16), then near 0,

$$
w(x)=2 \log |x|+o(1) .
$$

Proof We prove (3.17) by a blow-up argument. In a normal coordinate system at 0 , let $y=c_{m} x$ and $w_{m}(y)=w(x)+2 \log c_{m}$, where $c_{m}$ is any sequence converging to infinity. Let $\tilde{w}_{m}$ be the radial function corresponding to $w_{m}$. Then by (3.16),

$$
\tilde{w}_{m}(r)=2 \log r+O\left(c_{m}^{-1}\right) .
$$

Hence $\tilde{w}_{m} \rightarrow 2 \log r$.

For any fixed $r_{0}>0$ small, let $w_{m}\left(y_{m}\right)=\tilde{w}_{m}\left(r_{0}\right)\left(\left|y_{m}\right|=r_{0}\right)$. We may assume that $y_{m} \rightarrow y_{0}$. By the Hölder continuity (Corollary 3.2), we may also assume that in a neighborhood of $y_{0}$, $w_{m}$ converges uniformly to $w_{\infty}$. Then $w_{\infty}$ is a $k$-admissible function defined on $\mathbf{R}^{n}$. The comparison principle argument of Lemma 2.4 implies that $w_{\infty} \equiv 2 \log r$ in a neighborhood of $y_{0}$. The Hölder continuity in Corollary 3.2 implies that if $w_{\infty}=2 \log r$ at some point, $w_{\infty}$ is well-defined nearby. The comparison principle then implies that $w_{\infty} \equiv 2 \log r$ near the point. Hence $\tilde{w}_{m}$ converges uniformly to $w_{\infty}=2 \log r$ on $|x|=r_{0}$, and converges locally uniformly to $w_{\infty}=2 \log r$ in $\mathbf{R}^{n} \backslash\{0\}$. Hence (3.17) is proved.

Note that (3.17) is equivalent to (1.20), by the relation $v=e^{-\frac{n-2}{2} w}$.

Remark 3.2 From the proof of Lemma 3.3, we see that $w$ has only isolated singularities. For if there is a sequence of singular points $x_{m} \in \mathcal{M}$ which converges to a point 0 , we may choose $c_{m}=\left|x_{m}\right|^{-1}$ in the above argument. Then the limit function $w_{\infty}$ has at least two singular points 0 and $x^{*}=\lim x_{m} /\left|x_{m}\right|$. To see that $x^{*}$ is a singular point of the limit function $w_{\infty}$, we notice that the constant $C^{\prime}$ is uniformly bounded from above if $w$ is negative in a neighbourhood of 0 , which in turn implies that $\lim _{x \rightarrow x^{*}} w_{\infty}\left(x^{*}\right)=-\infty$. But the above argument shows that $w_{\infty}=2 \log r$. This is a contradiction.

Similarly one can show that the set of all isolated singular points has no limit points, for if there is a sequence of singular points $y_{m} \rightarrow 0$, we may for each $m>1$ choose a conformal normal coordinate with origin at $y_{m}$ and consider the sequence $x_{m}=y_{m+1}-y_{m}$. The above argument also leads to a contradiction.

Next we show that $w$ has at most one singular point.

Lemma 3.4 Let $w$ be a $k$-admissible function. Then the singularity set

$$
S_{w}=\bigcap_{h<0}\{x \in \mathcal{M} \mid w(x)<h\}
$$

contains at most one point.

Proof We adapt the corresponding argument in [18]. If $S_{w}$ is not empty, it consists of finitely many isolated points. Let $g=e^{-2 w} g_{0}$. By Lemma 3.3, $\left(\mathcal{M} \backslash S_{w}, g\right)$ is a complete manifold with finitely many ends. Now fixing a point $y \notin S_{w}$, we consider the ratio

$$
Q(r)=\frac{\operatorname{Vol}\left(B_{y, r}\right)}{r^{n}},
$$


where $B_{y, r}=B_{y, r}[g]$ is the geodesic ball of $(\mathcal{M}, g)$. By definition, as well as the local Hölder continuity (Lemma 3.1), there is a sequence of smooth $k$-admissible functions $w_{m}$ which converges to $w$ locally uniformly. It is easy to verify that for any fixed $y$ and $r$, $\operatorname{Vol}\left(B_{y, r}\left[g_{m}\right]\right) \rightarrow \operatorname{Vol}\left(B_{y, r}[g]\right)$ as $m \rightarrow \infty$, where $g_{m}=e^{-2 w_{m}} g_{0}$. Note that when $k \geq n / 2$ the Ricci curvature of $\left(\mathcal{M}, g_{m}\right)$ is positive, as shown in (1.8). Hence by the Bishop Theorem, the ratio $Q_{m}(r)=\operatorname{Vol}\left(B_{y, r}\left[g_{m}\right]\right) / r^{n}$ is decreasing for all $m$. Sending $m \rightarrow \infty$, we see that $Q$ is non-increasing in $r$. Hence

$$
Q(0) \leq \lim _{r \rightarrow 0} Q(r) \leq \frac{1}{n} \omega_{n},
$$

where $\omega_{n}$ is the area of the unit sphere $S^{n-1}$.

On the other hand, denote $A_{r_{1}, r_{2}}=B_{0, r_{2}}\left[g_{0}\right]-B_{0, r_{1}}\left[g_{0}\right]$, where $r_{2}>r_{1}>0$ are sufficiently small. We identify $A_{r_{1}, r_{2}}$ with the Euclidean annulus $A_{r_{1}, r_{2}}^{e}=\left\{x \in \mathbf{R}^{n} \mid r_{1}<\right.$ $\left.|x|<r_{2}\right\}$ by the exponential map. By the asymptotic behavior (3.17), the volume of $A_{r_{1}, r_{2}}$ in the metric $g=e^{-2 w} g_{0}$ is a lower order perturbation of that in the metric $g^{\prime}=e^{-2 w^{\prime}} g_{0}$, where $w^{\prime}=2 \log |x|$. But in our normal coordinates at 0 , by (3.2) the volume of $A_{r_{1}, r_{2}}$ in $g^{\prime}$ is the same as that of $A_{r_{1}, r_{2}}^{e}$ with the metric $g_{e}^{\prime}=e^{-2 w^{\prime}} g_{e}$, where $g_{e}$ is the standard Euclidean metric. Hence $\operatorname{Vol}_{g^{\prime}} A_{r_{1}, r_{2}}=\frac{1}{n} \omega_{n}\left(r_{1}^{-n}-r_{2}^{-n}\right)$. Therefore as $r \rightarrow \infty$, each end of the metric $g$ will contribute to the ratio $Q(r)$ a factor $\frac{1}{n} \omega_{n}$. Therefore we obtain

$$
\lim _{r \rightarrow \infty} Q(r)=\frac{m}{n} \omega_{n},
$$

where $m$ is the number of singular points of $w$. From (3.21) and (3.22) we see that if $S_{w}$ is not empty, then $m$ must be equal to 1 , namely $S_{w}$ is a single point.

\subsection{Smoothness of $k$-admissible functions}

In this subsection we prove the following smoothness result. The proof is again inspired by the corresponding proof in [18].

Lemma 3.5 Let $w$ be a $k$-admissible function $w$ with a singular point 0 . Then $w$ is $C^{\infty}$ smooth away from 0 .

Proof First we prove

$$
\sigma_{k}\left(\lambda\left(A_{g}\right)\right) \equiv 0 \quad \text { in } \mathcal{M} \backslash\{0\},
$$

where $g=e^{-2 w} g_{0}$. It suffices to prove that for any given point $x_{0} \neq 0$ and a sufficiently small $r>0\left(r<\frac{1}{4}\left|x_{0}\right|\right),(3.23)$ holds in $B_{x_{0}, r}=B_{x_{0}, r}\left[g_{0}\right]$.

By definition, there exists a sequence $w_{m}$ of smooth $k$-admissible functions which converges to $w$ in $B_{x_{0}, 2 r}$ uniformly. Let $\varphi_{m}$ be the admissible solution of the Dirichlet problem [12]

$$
\begin{aligned}
\sigma_{k}\left(\lambda\left(A_{g_{\varphi_{m}}}\right)\right)=\varepsilon_{m} & \text { in } B_{x_{0}, r}, \\
\varphi_{m}=w_{m} & \text { on } \partial B_{x_{0}, r},
\end{aligned}
$$

where $g_{\varphi_{m}}=e^{-2 \varphi_{m}} g_{0}$, and $\varepsilon_{m}$ is a small positive constant $\left(\varepsilon_{m} \rightarrow 0\right.$ as $\left.m \rightarrow \infty\right)$ such that $\sigma_{k}\left(\lambda\left(A_{g_{w_{m}}}\right)\right)>\varepsilon_{m}\left(g_{w_{m}}=e^{-2 w_{m}} g_{0}\right)$. By the comparison principle we have $\varphi_{m} \geq w_{m}$ in $B_{x_{0}, r}$. Let $\hat{w}_{m}=w_{m}$ in $\mathcal{M}-B_{x_{0}, r}$ and $\hat{w}_{m}=\varphi_{m}$ in $B_{x_{0}, r}$. Then $\hat{w}_{m}$ is $k$-admissible (see Corollary 3.8 below). Let $\hat{w}=\lim _{m \rightarrow \infty} \hat{w}_{m}$. Then $\hat{w}$ is a $k$-admissible function with 
singularity point 0 . Define the metric $\hat{g}=e^{-2 \hat{w}} g_{0}$ and the ratio $\hat{Q}(r)=\frac{\operatorname{Vol}\left(B_{y, r}[\hat{g}]\right)}{r^{n}}$. Then from the proof of Lemma 3.4, we also have $\hat{Q} \equiv \frac{1}{n} \omega_{n}$.

To prove (3.23) it suffices to show that $\hat{w} \equiv w$. Noting that $\hat{w}=w$ in $\mathcal{M}-B_{x_{0}, r}$ and $\hat{w} \geq w$ in $B_{x_{0}, r}$, we have $B_{y, r}[\hat{g}] \supset B_{y, r}[g]$ for any $r>0$ and $y \neq 0$. If there exists a point $y \in B_{x_{0}, r}$ such that $\hat{w}>w$ at $y$, then there exists a positive constant $\delta>0$ such that for any $r>1$,

$$
B_{y, r}[\hat{g}] \supset B_{y, r+\delta}[g] .
$$

But this is impossible as both the ratios $Q(r)$ and $\hat{Q}(r)$ are constant.

By the interior second order derivative estimate [13], we see that $w$ is $C^{1,1}$ smooth. Next we prove that $w$ is $C^{\infty}$ smooth away from 0 . By the regularity of linear elliptic equations [11], it suffices to prove that $v=e^{-\frac{n-2}{2} w} \in C^{1,1}$ is a strong solution to the uniformly elliptic equation

$$
-\Delta_{g_{0}} v+\frac{n-2}{4(n-1)} R_{g_{0}} v=0 \quad \text { in } \mathcal{M} \backslash\{0\},
$$

where $R_{g_{0}}$ is the scalar curvature of $\left(\mathcal{M}, g_{0}\right)$; namely the scalar curvature of $g=e^{-2 w} g_{0}$ vanishes identically.

Equation (3.25) may be verified as in Sect. 7.6 in [18]. Here we provide a proof for completeness. Since $w \in C^{1,1}$, it is twice differentiable almost everywhere. Suppose at a point $0, w$ is twice differentiable and the scalar curvature $R>0$. Then with respect to normal coordinates of $g$ at 0 , we have the expansion

$$
\operatorname{det} g_{i j}=1-\frac{1}{3} R_{i j} x_{i} x_{j}+o\left(|x|^{2}\right),
$$

see (5.2) in [19]. Hence

$$
\begin{aligned}
\operatorname{Vol}\left(B_{0, r}[g]\right) & =\int_{B_{0, r}} \sqrt{\operatorname{det} g_{i j}} \\
& =\int_{B_{0, r}}\left[1-\frac{1}{6} R_{i j} x_{i} x_{j}+o\left(|x|^{2}\right)\right] \\
& =\frac{1}{n} \omega_{n} r^{n}\left[1-\frac{R}{6(n+2)} r^{2}+o\left(r^{2}\right)\right],
\end{aligned}
$$

where $R_{i j}$ and $R$ are respectively the Ricci curvature and the scalar curvature in $g$. This is a contradiction when $R>0$ at 0 , as the ratio $Q$ is a constant. Hence the scalar curvature of $g$ vanishes almost everywhere.

\subsection{End of proof of Theorem A}

From Sects. 3.2 and 3.3, we see that if $\left(\mathcal{M}, g_{0}\right)$ is a compact manifold and there exists a $k$-admissible function $w$ with singularity at some point 0 , then $w$ has the asymptotic formula (3.17) and $w$ is smooth away from 0 . The manifold $\mathcal{M} \backslash\{0\}$ equipped with the metric $g=e^{-2 w} g_{0}$ is a complete manifold with nonnegative Ricci curvature, and satisfies furthermore the volume growth formula $Q(r) \equiv 1$. Hence $(\mathcal{M} \backslash\{0\}, g)$ is isometric to the Euclidean space [7]. Hence $\left(\mathcal{M}, g_{0}\right)$ is conformally equivalent to the unit sphere $S^{n}$. See also [18].

To finish the proof of Theorem A, it suffices to prove 
Lemma 3.6 Let $\left(\mathcal{M}, g_{0}\right)$ be a compact manifold. If $\left(\mathcal{M}, g_{0}\right)$ is not conformally equivalent to the unit sphere $S^{n}$, then there exists $K>0$ such that if $w$ is a $k$-admissible function,

$$
\begin{aligned}
& \sup _{\mathcal{M}} w-\inf _{\mathcal{M}} w \leq K, \\
& |w(x)-w(y)| \leq K|x-y|^{2-\frac{n}{k}} .
\end{aligned}
$$

Proof If (3.28) is not true, there exists a sequence of $k$-admissible functions $w_{m}$ such that $\sup _{\mathcal{M}} w_{m}=0$ (by subtracting a constant if necessary) and $\inf _{\mathcal{M}} w_{m} \rightarrow-\infty$. Suppose that $w_{m}(0) \rightarrow-\infty$. By Lemma 3.1, $\left\{e^{w_{m}}\right\}$ are uniformly Holder continuous. Hence we may assume that $e^{w_{m}}$ converges locally uniformly to $e^{w}$ in $\mathcal{M}$. The Hölder continuity also implies that $e^{w}(0)=0$, namely $\lim _{x \rightarrow 0} w(x)=-\infty$. By a weak Harnack inequality for (1.6), the set $\{w=-\infty\}$ has measure zero and so $w$ is $k$-admissible. Applying Lemma 3.3 to the limit function $w$ we conclude that $w$ has the asymptotic behavior (3.17) and that 0 is an isolated singularity of $w$ (see Remark 3.2). Therefore by Lemmas 3.4, 0 is the unique singular point of $w$. By Lemma 3.5, $w$ is $C^{\infty}$-smooth away from 0 . Hence as above, we see that $\left(\mathcal{M}, g_{0}\right)$ is conformally equivalent to the unit sphere $S^{n}$, which is ruled out by our assumption. Hence (3.28) holds.

The Hölder continuity (3.29) follows from Lemma 3.1.

\subsection{Remarks on the set $\left[g_{0}\right]_{k}$}

In this section we prove some properties of $k$-admissible functions.

Lemma 3.7 If $w_{1}, w_{2}$ are smooth and $k$-admissible, then $w=\max \left(w_{1}, w_{2}\right)$ is $k$-admissible.

Proof It is convenient to consider the function $u=e^{w}$. By approximation we suppose $u_{1}$ and $u_{2}$ are smooth and $k$-admissible functions such that the eigenvalues $\lambda(U)$ lie strictly in the open convex cone $\Gamma_{k}$, where $U$ is the matrix (1.16) with $u=u_{1}$ and $u_{2}$. Hence when $r>0$ is sufficiently small, the eigenvalues of the matrix

$$
U_{r}=\left\{u_{i j}-\frac{|\nabla u|^{2}}{2 u_{x, r}}+u A_{g_{0}}\right\}
$$

lie in $\Gamma_{k}$ for $u=u_{1}$ and $u_{2}$, where $u_{x_{0}, r}=\inf _{B_{x_{0}, r}} u$.

Let $u=\max \left(u_{1}, u_{2}\right)$. Since $u_{1}, u_{2}$ are smooth functions, $u$ is twice differentiable almost everywhere. Let $\rho \in C_{0}^{\infty}\left(\mathbf{R}^{n}\right)$ be a mollifier. In particular we choose $\rho$ to be a radial, smooth, nonnegative function, supported in the unit ball $B_{0,1}$, with $\int_{B_{0,1}} \rho=1$. Let

$$
u_{[\varepsilon]}(x)=\int_{B_{x, \varepsilon}} \varepsilon^{-n} \rho\left(\frac{|x-y|}{\varepsilon}\right) u(y) \sqrt{\operatorname{det}\left(g_{0}\right)_{i j}} d y
$$

be the mollification of $u$, where $B_{x, \varepsilon}$ is the geodesic ball. For each point $x$, using normal coordinates and the exponential map, we have, by (3.26),

$$
\begin{aligned}
u_{[\varepsilon]}(x) & =\int_{B_{0,1}} \rho(y) u(x-\varepsilon y) \sqrt{\operatorname{det}\left(g_{0}\right)_{i j}} d y \\
& =\int_{B_{0,1}} \rho(y) u(x-\varepsilon y)\left(1-\frac{\varepsilon^{2}}{6} R_{i j}(x) y_{i} y_{j}+O\left(\varepsilon^{3}\right)\right) d y,
\end{aligned}
$$


where $B_{0,1}$ is the Euclidean space. If $g_{0}$ is a flat metric, we have

$$
\begin{aligned}
\nabla u_{[\varepsilon]} & =\int_{B_{0,1}} \rho(y) \nabla u(x-\varepsilon y) d y, \\
\nabla^{2} u_{[\varepsilon]} & \geq \int_{B_{0,1}} \rho(y) \nabla^{2} u(x-\varepsilon y) d y, \\
\left|\nabla u_{[\varepsilon]}\right|^{2} & =\left[\int_{B_{0,1}} \rho(y) \nabla u(x-\varepsilon y) d y\right]^{2} \\
& \leq \int_{B_{0,1}} \rho(y)|\nabla u(x-\varepsilon y)|^{2} d y .
\end{aligned}
$$

Hence $u_{[\varepsilon]}$ is $k$-admissible by (3.30). If $g_{0}$ is not flat, by (3.32), an extra term of magnitude $O\left(\varepsilon^{2}\right)$ arises. Letting $\varepsilon>0$ be sufficiently small and noting that the eigenvalues of $U$ (with respect to $u_{1}$ and $u_{2}$ ) lie strictly in the open set $\Gamma_{k}$, we conclude again that $u_{[\varepsilon]}$ is $k$-admissible.

Corollary 3.8 Suppose $\varphi$ is a smooth $k$-admissible function on $\mathcal{M}$ with $\sigma_{k}\left(\lambda\left(A_{g_{\varphi}}\right)\right)>f$, where $g_{\varphi}=e^{-2 \varphi} g_{0} \in\left[g_{0}\right]_{k}$ and $f$ is a smooth, positive function. Let $w$ be the admissible solution of

$$
\begin{aligned}
\sigma_{k}(\lambda(W))=f & \text { in } \Omega, \\
w=\varphi & \text { on } \partial \Omega,
\end{aligned}
$$

where $W$ is given in (1.17), and $\Omega$ is a smooth domain on $\mathcal{M}$. Extend $w$ to $\mathcal{M}$ by letting $w=\varphi$ on $\mathcal{M}-\Omega$. Then $w$ is $k$-admissible.

It was proved in [12] that (3.36) admits a solution $w$, smooth up to the boundary. By the comparison principle we have $w>\varphi$ in $\Omega$ and $\partial_{\nu}(\varphi-w)>0$ on $\partial \Omega$, where $v$ is the unit outward normal. Hence we can extend $w$ to a neighbourhood of $\Omega$ such that it is $k$-admissible. Hence Corollary 3.8 follows from Lemma 3.7.

Corollary 3.9 Consider the Dirichlet problem (3.36). Suppose the set of sub-solutions $W_{\text {sub }}$ is not empty. Let

$$
w(x)=\sup \left\{\varphi(x) \mid \varphi \in W_{\text {sub }}\right\} .
$$

If $w$ is bounded from above, then it is a solution to (3.36).

By the interior a priori estimates [13], the proof is standard. Note that in Corollary 3.9, we allow $\Omega$ to be the whole manifold $\mathcal{M}$.

\section{Proof of Theorem C}

We divide the proof into three cases, according to $p<k, p=k$, and $p>k$.

Case $1 p<k$. By (1.15), we can write Eq. (1.12) as

$$
\sigma_{k}(\lambda(W))=f e^{a w},
$$


where $f$ is a constant multiple of that in (1.12),

$$
a=\frac{1}{2}(n-2)(k-p) .
$$

For any given $k$-admissible function $w$, the functions $w+c$ and $w-c$ are respectively a super and a sub solution of (4.1) provided the constant $c$ is sufficiently large. By the a priori estimates in [13] and the comparison principle, the solution of (4.1) is uniformly bounded. When $a>0$, the linearized equation of (4.1) is invertible. Hence by the continuity method, there is a unique smooth solution to (4.1).

Case $2 p=k$. We prove that for any positive smooth function $f$, there is a unique constant $\theta>0$ such that the equation

$$
\sigma_{k}(\lambda(W))=\theta f
$$

has a solution. For $a>0$ small, let $w_{a}$ be the solution of (4.1). Let $c_{a}=\inf w_{a}$. We write (4.1) in the form

$$
\sigma_{k}\left(\lambda\left(W_{a}\right)\right)=\left(f e^{a c_{a}}\right) e^{a\left(w_{a}-c_{a}\right)},
$$

where $W_{a}$ is the matrix (1.17) relative to $w_{a}$. Assume $g_{0} \in\left[g_{0}\right]_{k}$ so that $\lambda\left(A_{g_{0}}\right) \in \Gamma_{k}$. Then at the maximum point of $w_{a}$,

$$
\sigma_{k}\left(\lambda\left(A_{g_{0}}\right)\right) \geq \sigma_{k}\left(\lambda\left(W_{a}\right)\right) \geq f e^{a c_{a}} .
$$

At the minimum point of $w_{a}$,

$$
\sigma_{k}\left(\lambda\left(A_{g_{0}}\right)\right) \leq \sigma_{k}\left(\lambda\left(W_{a}\right)\right)=f e^{a c_{a}} .
$$

Hence $e^{a c_{a}}$ is strictly positive and uniformly bounded as $a \rightarrow 0$. By the a priori estimates [13], where the estimates depend only on $\inf \left(w_{a}-c_{a}\right)$, we see that $w_{a}-c_{a}$ is uniformly bounded from above and sub-converges to a solution $w_{0}$ of (4.3) with $\theta=\lim _{a \rightarrow 0} e^{a c_{a}}$. By the maximum principle it is easy to see that if $w^{\prime}$ is another solution, then necessarily $w^{\prime}=w_{0}+$ const; and furthermore (4.3) has no ( $k$-admissible) solution for different $\theta$.

Case $3 p>k$. In this case we adopt the degree argument from [30], see the proof of Theorem 5.1 there. Alternatively we can also use the degree argument in Sect. 3 of [30]. We will study the auxiliary problem

$$
\sigma_{k}(\lambda(V))=t\left(\delta_{t}+f v^{p}\right),
$$

where $t \geq 0$ is a parameter and $\delta_{t}$ is a positive constant depending on $t, \delta_{t}=\delta_{0} \leq 1$ when $t \leq 1$ and $\delta_{t}=1$ when $t>2$, and $\delta_{t}$ is smooth and monotone increasing when $1 \leq t \leq 2$.

Claim 1 For any $t_{0}>0$, the solution of (4.6) is uniformly bounded when $t \geq t_{0}$. Indeed, if there exists a sequence of solutions $\left(t_{j}, v_{j}\right)$ of (4.6) such that $t_{j} \geq t_{0}$ and $\sup v_{j} \rightarrow \infty$, we have $m_{j}=\inf v_{j} \rightarrow \infty$ by (1.5). The function $v_{j}^{\prime}=v_{j} / m_{j}$ satisfies

$$
\begin{aligned}
\sigma_{k}\left(\lambda\left(V^{\prime}\right)\right) & \left.\geq t_{j} f m_{j}^{p-k}\left(v_{j}^{\prime}\right)^{p}\right) \\
& \geq t_{j} f m_{j}^{p-k} \rightarrow \infty,
\end{aligned}
$$

where $V^{\prime}$ is the matrix (1.11) relative to $v^{\prime}$. From (4.7) and the comparison principle we have $\sup v_{j}^{\prime} \rightarrow \infty$. Hence inf $v_{j}^{\prime} \rightarrow \infty$ by (1.5), which contradicts to the definition of $v_{j}^{\prime}$. 
Define the mapping $T_{t}$ so that for any $v_{1} \in C^{2}(\mathcal{M}), T_{t}\left(v_{1}\right)$ is the solution of

$$
\sigma_{k}(\lambda(V))=t\left(\delta_{t}+f v_{1}^{p}\right) .
$$

Then a solution of (4.6) is a fixed point of $T_{t}$.

Claim 2 There is a solution of (4.6) when $t>0$ is small. Indeed, for any smooth, positive function $\varphi^{*}$, denote $\Phi=\left\{\varphi \in C^{2}(\mathcal{M}) \mid 0<\varphi<\varphi^{*},\|\varphi\|_{C^{2}}<R\right\}$ for some large constant $R>0$. Then when $\delta_{0}, t>0$ are small, $T(\Phi)$ is strictly contained in $\Phi$. Hence the degree $\operatorname{deg}\left(I-T_{t}, \Phi, 0\right)$ is well defined for $t>0$ small. Extend $T_{t}$ to $t=0$ by letting $T_{t}(v)=0$ for all $v$, so that $T_{t}$ is also continuous at $t=0$. Hence

$$
\operatorname{deg}\left(I-T_{t}, \Phi, 0\right)=\operatorname{deg}\left(I-T_{0}, \Phi, 0\right)=1 .
$$

Hence $T_{t}$ has a fixed point in $\Phi$ for $t>0$ small.

Note that when $\delta_{0}$ is sufficiently small, letting $\varphi^{*}$ be a small positive constant, we see that (4.6) has a solution for $t \leq 1$.

Claim 3 Let $t^{*}=\sup \left\{t \mid\right.$ (4.6) admits a solution\}. Then $t^{*}$ is finite. Indeed, if $t^{*}=\infty$, there is a sequence $t_{j} \rightarrow \infty$ such that (4.6) has a solution $v_{j}$. We have obviously $m_{j}=\inf v_{j} \rightarrow \infty$, which is a contradiction with Claim 1.

Claim 4 Equation (4.6) has a solution at $t=t^{*}$. Indeed, let $t_{j} \nearrow t^{*}$ and $v_{j}$ be the corresponding solution of (4.6). By claim 1, $v_{j}$ is uniformly bounded. Hence $v_{j}$ sub-converges to a solution $v^{*}$ of (4.6) with $t=t^{*}$.

Now we choose $\varphi^{*}=v^{*}$ and define $\Phi$ as above. For any $v_{1} \in \Phi$, let $v$ be the solution of (4.8). Since for any $t \in\left(0, t^{*}\right), v^{*}$ is a super-solution of (4.6). We have $0<v<v^{*}$ by the maximum principle. Hence by (4.9), $\operatorname{deg}\left(I-T_{t}, \Phi, 0\right)=1$ for $t \in\left[0, t^{*}\right)$.

On the other hand, for any given $t_{0}>0$, since the solution of (4.6) is uniformly bounded for $t \geq t_{0}$, the degree $\operatorname{deg}\left(I-T_{t}, B_{R}, 0\right)$ is well defined for $t \in\left(t_{0}, t^{*}+1\right]$ for sufficiently large $R$, where $B_{R}=\left\{v \in C^{2}(\mathcal{M}) \mid 0<v<R,\|v\|_{C^{2}}<R\right\}$. But when $t>t *$, (4.6) has no solution. Hence $\operatorname{deg}\left(I-T_{t}, B_{R}, 0\right)=0$. Hence for any $t \geq t_{0}$, (4.6) has a solution $v \notin \Phi$ with degree -1 .

Let $v=v_{\delta_{0}} \notin \Phi$ be a solution of (4.6) at $t=1$. We have $\sup v>\inf v^{*}>0$. Let $\delta_{0} \rightarrow 0$. Since the solution is uniformly bounded, it converges to a solution of (1.12). This completes the proof.

From the above argument, we have the following extensions.

Corollary 4.1 Let $\left(\mathcal{M}, g_{0}\right)$ be a compact $n$-manifold not conformally equivalent to the unit sphere $S^{n}$. Suppose $\frac{n}{2}<k \leq n$ and $\left[g_{0}\right]_{k} \neq \emptyset$. Suppose there exists a constant $c_{0}>0$ such that

$$
\begin{aligned}
\varphi(x, t) & \geq c_{0}, \\
\lim _{t \rightarrow \infty} t^{-k} \varphi(x, t) & =\infty .
\end{aligned}
$$

Then there exists a constant $t^{*}>0$ such that the equation

$$
\sigma_{k}(\lambda(V))=t \varphi(x, v)
$$

has at least two solutions for $0<t<t^{*}$, one solution at $t=t^{*}$, and no solution for $t>t^{*}$. 
Corollary 4.2 Let $\left(\mathcal{M}, g_{0}\right)$ be as in Corollary $4.1, \frac{n}{2}<k \leq n$. Suppose $\varphi>0$,

$$
\lim _{t \rightarrow 0} t^{-k} \varphi(x, t)=0,
$$

and (4.11) holds. Then there exists a solution to (1.10).

In the above theorems, we can also allow that the right hand side depends on the gradient $\nabla v$. Furthermore, (4.11) and (4.13) can be relaxed to

$$
\begin{aligned}
& \lim _{t \rightarrow \infty} t^{-k} \varphi(x, t)>\theta, \\
& \lim _{t \rightarrow 0} t^{-k} \varphi(x, t)<\theta,
\end{aligned}
$$

where $\theta$ is the eigenvalue of (1.13) (with $f \equiv 1$ ). See [30] for the Monge-Ampère equation.

We remark that when $1 \leq k \leq \frac{n}{2}$, Theorem $\mathrm{C}$ holds for $p<k \frac{n+2}{n-2}$. Indeed, when $p \leq k$, the proof of the Cases 1 and 2 above also applies to the cases $1 \leq k \leq \frac{n}{2}$. When $k<p<k \frac{n+2}{n-2}$, by a blow-up argument and the Liouville theorem [20], it is known that the set of solutions to (4.6) is uniformly bounded. Hence by the above degree argument, one also obtains the existence of solutions.

Corollary 4.3 Let $\left(\mathcal{M}, g_{0}\right)$ be a compact $n$-manifold with $\left[g_{0}\right]_{k} \neq \emptyset, 1 \leq k \leq n$. Then for any smooth, positive function $f$ and any constant $p \neq k, p<k \frac{n+2}{n-2}$, there exists a positive solution to the (1.12). The solution is unique if $p<k$. When $p=k$, there exists a unique constant $\theta>0$ such that (1.13) has a solution. The solution is unique up to a constant multiplication.

Note that in Corollary 4.3 we allow that $\left(\mathcal{M}, g_{0}\right)$ is the unit sphere.

\section{References}

1. Aubin, T.: Equations différentielles non linéaires et problìe de Yamabe concernant la courbure scalaire. J. Math. Pures Appl. 55, 269-296 (1976)

2. Aubin, T.: Some nonlinear problems in Riemannian geometry. Springer, Heidelberg (1998)

3. Caffarelli, L.A., Nirenberg, L., Spruck, J.: Dirichlet problem for nonlinear second order elliptic equations III. Functions of the eigenvalues of the Hessian. Acta Math. 155, 261-301 (1985)

4. Cao, J.-G.: The existence of generalized isothermal coordinates for higher-dimensional Riemannian manifolds. Trans. Am. Math. Soc. 324, 901-920 (1991)

5. Chang, A., Gursky, M., Yang, P.: An equation of Monge-Ampère type in conformal geometry, and four-manifolds of positive Ricci curvature. Ann. Math. 155(2), 709-787 (2002)

6. Chang, A., Gursky, M., Yang, P.: An a priori estimate for a fully nonlinear equation on four-manifolds. J. Anal. Math. 87, 151-186 (2002)

7. Chavel, I.: Riemannian geometry - a modern introduction. Cambridge Univ. Press, London (1993)

8. Chou, K.S. (Tso, K.): On a real Monge-Ampère functional. Invent. Math. 101 425-448 (1990)

9. Chou, K.S., Wang, X.J.: A variational theory of the Hessian equation. Comm. Pure Appl. Math. 54, 1029-1064 (2001)

10. Ge, Y., Wang, G.: On a fully nonlinear Yamabe problem. Ann. Sci. École Norm. Sup. 39(4), 569-598 (2006)

11. Gilbarg, D., Trudinger, N.S.: Elliptic partial differential equations of second order. Springer, Heidelberg (1983)

12. Guan, B.: Conformal metrics with prescribed curvature functions on manifolds with boundary. Am. J. Math. 129, 915-942 (2007)

13. Guan, P., Wang, G.: Local estimates for a class of fully nonlinear equations arising from conformal geometry. Int. Math. Res. Not. (26), 1413-1432 (2003)

14. Guan, P., Wang, G.: A fully nonlinear conformal flow on locally conformally flat manifolds. J. Reine Angew. Math. 557, 219-238 (2003) 
15. Guan, P., Viaclovsky, J., Wang, G.: Some properties of the Schouten tensor and applications to conformal geometry. Trans. Am. Math. Soc. 355, 925-933 (2003)

16. Günther, M.: Conformal normal coordinates. Ann. Global Anal. Geom. 11, 173-184 (1993)

17. Gursky, M., Viaclovsky, J.: Convexity and singularities of curvature equations in conformal geometry, Int. Math. Res. Not., Art. ID 96890, pp. 43 (2006)

18. Gursky, M., Viaclovsky, J.: Prescribing symmetric functions of the eigenvalues of the Ricci tensor. Ann. Math. 166(2), 475-531 (2007)

19. Lee, J.M., Parker, T.H.: The Yamabe problem. Bull. Am. Math. Soc. 17, 37-91 (1987)

20. Li, A., Li, Y.Y.: On some conformally invariant fully nonlinear equations. Comm. Pure Appl. Math. 56, 1416-1464 (2003)

21. Li, A., Li, Y.Y.: On some conformally invariant fully nonlinear equations II, Liouville, Harnack, and Yamabe. Acta Math. 195, 117-154 (2005)

22. Schoen, R.: Conformal deformation of a Riemannian metric to constant scalar curvature. J. Differ. Geom. 20, 479-495 (1984)

23. Schoen, R., Yau, S.T.: Lectures on Differential geometry. International Press (1994)

24. Sheng, W.M., Trudinger, N.S., Wang, X.-J.: The Yamabe problem for higher order curvatures. J. Differ. Geom. 77, 515-553 (2007)

25. Trudinger, N.S.: On Harnack type inequalities and their application to quasilinear elliptic equations. Comm. Pure Appl. Math. 20, 721-747 (1967)

26. Trudinger, N.S.: Remarks concerning the conformal deformation of Riemannian structures on compact manifolds. Ann. Scuola Norm. Sup. Pisa 22(3), 265-274 (1968)

27. Trudinger, N.S., Wang, X.J.: Hessian measures II. Ann. Math. 150(2), 579-604 (1999)

28. Trudinger, N.S., Wang, X.-J.: The intermediate case of the higher order Yamabe problem, (in press)

29. Viaclovsky, J.: Conformal geometry, contact geometry, and the calculus of variations. Duke Math. J. 101(2), 283-316 (2000)

30. Wang, X.-J.: Existence of multiple solutions to the equations of Monge-Ampère type. J. Differ. Eqs. 100, 95-118 (1992) 